\title{
Analysis of a stochastic single species model with Allee effect and jump-diffusion
}

Yalin $\operatorname{Jin}^{1 *}$

\footnotetext{
"Correspondence: hitylj@163.com ${ }^{1}$ Faculty of Chemical Engineering, Huaiyin Institute of Technology, Huaian, P.R. China
}

\begin{abstract}
In this paper, we consider the effects of the small and abrupt random perturbations in the environment, and formulate a stochastic single species model with Allee effect and jump-diffusion. We first prove that the model admits a unique solution which is global and positive. Then we study the stochastic permanence and extinction of the model. In addition, we estimate the growth rate of the solution. Our results reveal that the properties of the model have close relationships with the jump-diffusion. Finally, we work out several numerical simulations to validate the theoretical results.
\end{abstract}

MSC: $60 \mathrm{H} 10 ; 60 \mathrm{H} 30 ; 92 \mathrm{D} 25$

Keywords: Allee effect; Lévy jumps; Extinction; Stochastic permanence

\section{Introduction}

In the natural world, plenty of species exhibit the Allee effect [7], for instance, meerkats [6] and the African wild dogs [4]. In order to describe the Allee effect, a lot of mathematical equations have been developed and studied, see, e.g., [5, 9-11, 14, 23]. In particular, Dennis [9], Stephens and Sutherland [23], Kang and Udiani [14] considered the following single species model with Allee effect:

$$
\frac{\mathrm{d} \psi(t)}{\mathrm{d} t}=\psi(t)\left[r-\beta_{1} \psi(t)\right]-\frac{\beta_{2} \psi(t)}{\beta_{2} \alpha \psi(t)+1},
$$

where

$$
\begin{aligned}
& \psi(t) \text { population size, } \\
& r \text { intrinsic growth rate, }
\end{aligned}
$$

$\beta_{1}>0$ intraspecific competition rate,

$\beta_{2}>0$ attack rate,

$\alpha>0$ handling time of predator.

On the other hand, the growth of populations often encounters environmental perturbations. Therefore, one should introduce stochastic factors into population models $[8,9,12,13,18,26]$. In $[9,26]$ the authors researched the following stochastic single species

(c) The Author(s) 2020. This article is licensed under a Creative Commons Attribution 4.0 International License, which permits use, sharing, adaptation, distribution and reproduction in any medium or format, as long as you give appropriate credit to the original author(s) and the source, provide a link to the Creative Commons licence, and indicate if changes were made. The images or other third party material in this article are included in the article's Creative Commons licence, unless indicated otherwise in a credit line to the material. If material is not included in the article's Creative Commons licence and your intended use is not permitted by statutory regulation or exceeds the permitted use, you will need to obtain permission directly from the copyright holder. To view a copy of this licence, visit http://creativecommons.org/licenses/by/4.0/. 
model with Allee effect driven by the Brownian motion:

$$
\mathrm{d} \psi(t)=\psi(t)\left[r-\beta_{1} \psi(t)-\frac{\beta_{2}}{\beta_{2} \alpha \psi(t)+1}\right] \mathrm{d} t+\xi \psi(t) \mathrm{d} B(t),
$$

where $B(t)$ stands for a standard Brownian motion defined on a completed probability space $(\Omega, \mathcal{F}, \mathbb{P}), \xi$ represents the intensity of the stochastic perturbations. The authors of [26] investigated the persistence, extinction, stochastic permanence and the ergodicity of model (2).

Nevertheless, there are lots of abrupt perturbations in the environment which cannot be characterized by the Brownian motion, for instance, drought, epidemic, pesticides. Several scholars (see $[2,3,8,19,20]$ ) have suggested that one might utilize a Lévy jump process to characterize these abrupt perturbations. Hence, Eq. (2) is replaced by

$$
\begin{aligned}
\mathrm{d} \psi(t)= & \psi\left(t^{-}\right)\left[r-\beta_{1} \psi\left(t^{-}\right)-\frac{\beta_{2}}{\beta_{2} \alpha \psi\left(t^{-}\right)+1}\right] \mathrm{d} t+\xi \psi\left(t^{-}\right) \mathrm{d} B(t) \\
& +\int_{\mathbb{X}} \theta(x) \psi\left(t^{-}\right) \tilde{\Upsilon}(\mathrm{d} t, \mathrm{~d} x)
\end{aligned}
$$

where $\psi\left(t^{-}\right)$is the left limit of $\psi(t), \mathbb{X} \subset(0,+\infty), \widetilde{\Upsilon}(\mathrm{d} t, \mathrm{~d} x)=\Upsilon(\mathrm{d} t, \mathrm{~d} x)-\pi(\mathrm{d} x) \mathrm{d} t, \Upsilon$ represents a Poisson counting measure, $\pi$ stands for the characteristic measure of $\Upsilon$ with $\pi(\mathbb{X})<+\infty$. To the best of our current knowledge, there are no studies of Eq. (3).

In this paper we explore model (3). We will prove in Sect. 2 that the model admits a unique solution which is global and positive. Then we study the stochastic permanence and extinction of the model in Sect. 3. In Sect. 4, we estimate the growth rate of the solution. In Sect. 5, we work out several simulations to numerically illustrate that the properties of the model have close relationships with the jump-diffusion.

\section{The existence and uniqueness of the solution}

Let $C_{1}, C_{2}, \ldots, C_{7}$ be positive constants. Throughout this article, we assume that $\Upsilon$ and $B(t)$ are independent, $1+\theta(x)>0, \forall u \in \mathbb{X}$, and

(H) $\int_{\mathbb{X}}[\ln (1+\theta(x))]^{2} \pi(\mathrm{d} x)<C_{1}$. This hypothesis illustrates that the jumps are tempered.

Equation (3) is a population system, we must illustrate that it admits a unique global positive solution for any given positive initial value. To prove this, we need to recall a lemma. Let $Y(t, y)$ represent the solution of the following scalar equation with initial value $Y(0)=y \neq 0$ :

$$
\mathrm{d} Y(t)=G_{1}\left(Y\left(t^{-}\right)\right) \mathrm{d} t+G_{2}\left(Y\left(t^{-}\right)\right) \mathrm{d} B(t)+\int_{\mathbb{X}} G_{3}\left(\left(Y\left(t^{-}\right), x\right) \tilde{\Upsilon}(\mathrm{d} t, \mathrm{~d} x),\right.
$$

where $G_{1}(0)=G_{2}(0)=0$, and $G_{3}(0, x)=0$ for $\forall x \in \mathbb{X} ; G_{1}, G_{2}, G_{3}$ are locally Lipschitz continuous and measurable functions. Define

$$
\Lambda(y)=\int_{\mathbb{X}}\left(\ln \frac{\left|y+G_{3}(y, x)\right|}{|y|}\right)^{2} \pi(\mathrm{d} x) .
$$

Lemma 1 ([25]) If for every $n \geq 1$,

$$
\sup _{0<|y| \leq n} \Lambda(y)<\infty
$$


then

$$
\mathrm{P}\left(Y(t, y) \neq 0 \text { and } Y\left(t^{-}, y\right) \neq 0, \forall t \geq 0\right)=1 \text {. }
$$

Theorem 1 For arbitrary initial data $\psi(0)>0$, model (3) admits a unique solution $\psi(t)$ which is global and positive almost surely (a.s.).

Proof Since the coefficients of Eq. (3) are locally Lipschitz, for arbitrary $\psi(0)>0$, Eq. (3) admits a unique local solution $\psi(t)$ for $t \in\left[0, \tau_{e}\right)$, where $\tau_{e}$ is the explosion time (see [3]). Notice that for $t \in\left[0, \tau_{e}\right)$,

$$
\begin{aligned}
\psi(t)= & \psi(0) \exp \left\{r t-\frac{\xi^{2}}{2} t-\beta_{1} \int_{0}^{t} \psi(s) \mathrm{d} s-\int_{0}^{t} \frac{\beta_{2}}{\beta_{2} \alpha \psi(s)+1} \mathrm{~d} s+\xi B(t)\right. \\
& \left.-t \int_{\mathbb{X}}(\theta(x)-\ln (1+\theta(x))) \pi(\mathrm{d} x)+\int_{0}^{t} \int_{\mathbb{X}} \ln (1+\theta(x)) \widetilde{\Upsilon}(\mathrm{d} s, \mathrm{~d} x)\right\} .
\end{aligned}
$$

Therefore for $t \in\left[0, \tau_{e}\right), \psi(t) \geq 0$. In addition, it follows from Hypothesis $(\mathrm{H})$ that the equation in (3) obeys (5). In view of Lemma $1, \psi(t)>0$ for $t \in\left[0, \tau_{e}\right)$.

We are in the position to show that $\tau_{e}=+\infty$. Let $k_{0}>0$ be a sufficiently large positive integer such that $\psi(0)<k_{0}$. For every $k>k_{0}$, define

$$
\tau_{k}:=\inf \left\{t \in\left[0, \tau_{e}\right): \psi(t)>k\right\} .
$$

Define $\tau_{\infty}:=\lim _{k \rightarrow+\infty} \tau_{k}$. Then $\tau_{\infty} \leq \tau_{e}$ a.s. Define $V(\psi)=\psi^{q}, 0<q<1$. Making use of Itô's formula (see [15]) yields that

$$
\mathbb{E}\left(\psi^{q}(t)\right)=\psi(0)+\mathbb{E} \int_{0}^{t} \mathcal{L} V(\psi(s)) \mathrm{d} s,
$$

where

$$
\begin{aligned}
\mathcal{L} V(\psi)= & q\left[r-\beta_{1} \psi-\frac{\beta_{2}}{\beta_{2} \alpha \psi+1}-(1-q) \xi^{2} / 2\right] \psi^{q} \\
& +\int_{\mathbb{X}}\left[(1+\theta(x))^{q}-1-q \theta(x)\right] \pi(\mathrm{d} x) \psi^{q} .
\end{aligned}
$$

Notice that

$$
u^{a} \leq 1+a(u-1), \quad u \geq 0,0<a<1,
$$

therefore,

$$
\begin{aligned}
\mathcal{L} V(\psi)= & q\left[r-\beta_{1} \psi-\frac{\beta_{2}}{\beta_{2} \alpha \psi+1}-(1-q) \xi^{2} / 2\right] \psi^{q} \\
& +\int_{\mathbb{X}}\left[(1+\theta(x))^{q}-1-q \theta(x)\right] \pi(\mathrm{d} x) \psi^{q} \\
\leq & q\left[r-\beta_{1} \psi\right] \psi^{q} \leq C_{2} .
\end{aligned}
$$


For $k>0$, define

$$
\sigma(k)=\inf \{V(\psi),|\psi| \geq k\} .
$$

Consequently,

$$
\lim _{k \rightarrow+\infty} \sigma(k)=+\infty
$$

Thus for any $T>0$,

$$
\sigma(k) \mathrm{P}\left(\tau_{k} \leq T\right) \leq \mathbb{E}\left(V\left(\psi\left(\tau_{k}\right)\right) I_{\left\{\tau_{k} \leq T\right\}}\right) \leq \mathbb{E}\left(V\left(\psi\left(\tau_{k} \wedge T\right)\right) \leq C_{3} .\right.
$$

Letting $k \rightarrow+\infty$ results in $\mathrm{P}\left(\tau_{\infty} \leq T\right)=0$. It then follows from the arbitrariness of $T$ that $\tau_{\infty}=\infty$. In other words, $\tau_{e}=\infty$.

\section{Permanence and extinction}

Now we explore the permanence and extinction of model (3).

Definition 1 ([2]) If for $\forall \varepsilon>0$, there are two positive constants $\kappa_{1}=\kappa_{1}(\varepsilon)$ and $\kappa_{2}=\kappa_{2}(\varepsilon)$ such that for $\forall \psi(0)>0$,

$$
\liminf _{t \rightarrow+\infty} \mathrm{P}\left\{\psi(t) \leq \kappa_{1}\right\} \geq 1-\varepsilon
$$

and

$$
\liminf _{t \rightarrow+\infty} \mathrm{P}\left\{\psi(t) \geq \kappa_{2}\right\} \geq 1-\varepsilon,
$$

then Eq. (3) is said to be stochastically permanent (SP).

Theorem 2 If $\mu>0$, then model (3) is $S P$, where

$$
\mu=r-\beta_{2}-\frac{\xi^{2}}{2}-\int_{\mathbb{X}}(\theta(x)-\ln (1+\theta(x))) \pi(\mathrm{d} x) .
$$

Proof To begin with, we claim that (8) holds. According to Itô's formula, we have

$$
\mathbb{E}\left(e^{t} \psi^{q}(t)\right)=\psi(0)+\mathbb{E} \int_{0}^{t} e^{s}\left[\psi^{q}(s)+\mathcal{L} V(\psi(s))\right] \mathrm{d} s .
$$

It is easy to see that $\psi^{q}+\mathcal{L} V(\psi) \leq\left[1+q r-\beta_{1} q \psi\right] \psi^{q}$, as a result,

$$
\mathbb{E}\left(e^{t} \psi^{q}(t)\right) \leq \psi(0)+C_{4} \int_{0}^{t} e^{s} \mathrm{~d} s=\psi(0)+C_{4}\left(e^{t}-1\right) .
$$

That is to say,

$$
\limsup _{t \rightarrow+\infty} \mathbb{E}\left(\psi^{q}(t)\right) \leq C_{4}
$$


For $\forall \varepsilon>0$, let $\kappa_{1}=C_{4}^{1 / q} / \varepsilon^{1 / q}$. Then Chebyshev's inequality (see, e.g., [21]) indicates that

$$
\mathrm{P}\left\{\psi(t)>\kappa_{1}\right\}=\mathrm{P}\left\{\psi^{q}(t)>\kappa_{1}^{q}\right\} \leq \mathbb{E}\left[\psi^{q}(t)\right] / \kappa_{1}^{q}=\kappa_{1}^{-q} \mathbb{E}\left[\psi^{q}(t)\right]
$$

In other words, $\limsup _{t \rightarrow+\infty} \mathrm{P}\left\{\psi(t)>\kappa_{1}\right\} \leq \varepsilon$, and so we obtain (8).

Now we claim that (9) holds. Define $V_{1}(\psi)=\psi^{-1}, \psi>0$. By Itô's formula, one obtains

$$
\begin{aligned}
\mathrm{d} V_{1}(\psi)= & -\frac{1}{\psi^{2}} \psi\left[\left(r-\beta_{1} \psi-\frac{\beta_{2}}{\beta_{2} \alpha \psi+1}\right) \mathrm{d} t+\xi \mathrm{d} B(t)\right]+\frac{1}{\psi^{3}} \xi^{2} \psi^{2} \mathrm{~d} t \\
& +\int_{\mathbb{X}}\left(\frac{1}{(1+\theta(x)) \psi}-\frac{1}{\psi}+\frac{1}{\psi^{2}} \theta(x) \psi\right) \pi(\mathrm{d} x) \mathrm{d} t \\
& +\int_{\mathbb{X}}\left(\frac{1}{(1+\theta(x)) \psi}-\frac{1}{\psi}\right) \tilde{\Upsilon}(\mathrm{d} t, \mathrm{~d} x) \\
= & V_{1}(\psi)\left[-r+\beta_{2}+\xi^{2}+\int_{\mathbb{X}}\left(\frac{1}{1+\theta(x)}-1+\theta(x)\right) \pi(\mathrm{d} x)\right] \mathrm{d} t \\
& +\beta_{1} \mathrm{~d} t-\frac{\beta_{2}^{2} \alpha}{\beta_{2} \alpha \psi+1} \mathrm{~d} t-\xi V_{1}(\psi) \mathrm{d} B(t)+V_{1}(\psi) \int_{\mathbb{X}}\left(\frac{1}{1+\theta(x)}-1\right) \tilde{\Upsilon}(\mathrm{d} t, \mathrm{~d} x) .
\end{aligned}
$$

Clearly,

$$
\begin{aligned}
\lim _{\lambda \rightarrow 0^{+}}\left\{\frac{\lambda \xi^{2}}{2}+\int_{\mathbb{X}}\left[\frac{1}{\lambda(1+\theta(x))^{\lambda}}-\frac{1}{\lambda}\right] \pi(\mathrm{d} x)\right\} & =\int_{\mathbb{X}} \ln \left(\frac{1}{1+\theta(x)}\right) \pi(\mathrm{d} x) \\
& =-\int_{\mathbb{X}} \ln (1+\theta(x)) \pi(\mathrm{d} x)
\end{aligned}
$$

Since $\mu>0$, there is a constant $\lambda>0$ such that

$$
r-\beta_{2}-\frac{\xi^{2}}{2}-\int_{\mathbb{X}} \theta(x) \pi(\mathrm{d} x)-\left\{\frac{\lambda \xi^{2}}{2}+\int_{\mathbb{X}}\left[\frac{1}{\lambda(1+\theta(x))^{\lambda}}-\frac{1}{\lambda}\right] \pi(\mathrm{d} x)\right\}>0
$$

Define $V_{2}(\psi)=V_{1}^{\lambda}(\psi)$. It then follows from Itô's formula that

$$
\begin{aligned}
\mathrm{d} V_{2}(t) & \\
= & \lambda V_{1}^{\lambda-1}(\psi)\left\{V_{1}(\psi)\left[-r+\beta_{2}+\xi^{2}+\int_{\mathbb{X}}\left(\frac{1}{1+\theta(x)}-1+\theta(x)\right) \pi(\mathrm{d} x)\right]\right. \\
& \left.+\beta_{1}-\frac{\beta_{2}^{2} \alpha}{\beta_{2} \alpha \psi+1}\right\} \mathrm{d} t \\
& +0.5 \lambda(\lambda-1) V_{1}^{\lambda-2}(\psi) \xi^{2} V_{1}^{2}(\psi) \mathrm{d} t-\lambda V_{1}^{\lambda-1}(\psi) \xi V_{1}(\psi) \mathrm{d} B(t) \\
& +\int_{\mathbb{X}}\left\{\left[V_{1}(\psi)+V_{1}(\psi)\left(\frac{1}{1+\theta(x)}-1\right)\right]^{\lambda}-V_{1}^{\lambda}(\psi)\right. \\
& \left.-V_{1}(\psi)\left(\frac{1}{1+\theta(x)}-1\right) \lambda V_{1}^{\lambda-1}(\psi)\right\} \pi(\mathrm{d} x) \mathrm{d} t \\
& +\int_{\mathbb{X}}\left\{\left[V_{1}(\psi)+V_{1}(\psi)\left(\frac{1}{1+\theta(x)}-1\right)\right]^{\lambda}-V_{1}^{\lambda}(\psi)\right\} \tilde{\Upsilon}(\mathrm{d} t, \mathrm{~d} x) \\
= & \lambda V_{1}^{\lambda-1}(\psi)\left\{-V_{1}(\psi)\left[r-\beta_{2}-0.5 \xi^{2}-\int\left(\frac{1}{1+\theta(x)}-1+\theta(x)\right) \pi(\mathrm{d} x)-0.5 \lambda \xi^{2}\right]\right.
\end{aligned}
$$




$$
\begin{aligned}
& \left.+\beta_{1}-\frac{\beta_{2}^{2} \alpha}{\beta_{2} \alpha \psi+1}\right\} \mathrm{d} t+V_{1}^{\lambda}(\psi) \int_{\mathbb{X}}\left[\frac{1}{(1+\theta(x))^{\lambda}}-1-\lambda\left(\frac{1}{1+\theta(x)}-1\right)\right] \pi(\mathrm{d} x) \mathrm{d} t \\
& -\lambda \xi V_{1}^{\lambda}(\psi) \mathrm{d} B(t)+V_{1}^{\lambda}(\psi) \int_{\mathbb{X}}\left[\frac{1}{(1+\theta(x))^{\lambda}}-1\right] \tilde{\Upsilon}(\mathrm{d} t, \mathrm{~d} x) \\
= & \lambda V_{1}^{\lambda-1}(\psi)\left\{-V_{1}(\psi)\left[r-\beta_{2}-0.5 \xi^{2}-\int_{\mathbb{X}} \theta(x) \pi(\mathrm{d} x)-0.5 \lambda \xi^{2}\right.\right. \\
& \left.-\int_{\mathbb{X}}\left(\frac{1}{\lambda(1+\theta(x))^{\lambda}}-\frac{1}{\lambda}\right) \pi(\mathrm{d} x)\right] \\
& \left.+\beta_{1}-\frac{\beta_{2}^{2} \alpha}{\beta_{2} \alpha \psi+1}\right\} \mathrm{d} t-\lambda \xi V_{1}^{\lambda}(\psi) \mathrm{d} B(t)+V_{1}^{\lambda}(\psi) \int_{\mathbb{X}}\left[\frac{1}{(1+\theta(x))^{\lambda}}-1\right] \tilde{\Upsilon}(\mathrm{d} t, \mathrm{~d} x) .
\end{aligned}
$$

Define $V_{3}(\psi)=e^{\theta t} V_{2}(\psi)$, where $\theta>0$ is a sufficiently small constant satisfying

$$
r-\beta_{2}-\xi^{2} / 2-\int_{\mathbb{X}} \theta(x) \pi(\mathrm{d} x)-\left\{\lambda \xi^{2} / 2+\int_{\mathbb{X}}\left[\frac{1}{\lambda(1+\theta(x))}-\frac{1}{\lambda}\right] \pi(\mathrm{d} x)\right\}>\theta / \lambda .
$$

Making use of Itô's formula leads to

$$
\begin{aligned}
\mathrm{d} V_{3}(t)= & \theta e^{\theta t} V_{1}^{\lambda}(\psi) \mathrm{d} t+\lambda e^{\theta t} V_{1}^{\lambda-1}(\psi)\left\{-V_{1}(\psi)\left[r-\beta_{2}-\xi^{2} / 2-\int_{\mathbb{X}} \theta(x) \pi(\mathrm{d} x)-\lambda \xi^{2} / 2\right.\right. \\
& \left.\left.-\int_{\mathbb{X}}\left(\frac{1}{\lambda(1+\theta(x))^{\lambda}}-\frac{1}{\lambda}\right) \pi(\mathrm{d} x)\right]+\beta_{1}-\frac{\beta_{2}^{2} \alpha}{\beta_{2} \alpha \psi+1}\right\} \mathrm{d} t \\
& -\lambda \xi e^{\theta t} V_{1}^{\lambda}(\psi) \mathrm{d} B(t)+e^{\theta t} V_{1}^{\lambda}(\psi) \int_{\mathbb{X}}\left[\frac{1}{(1+\theta(x))^{\lambda}}-1\right] \tilde{\Upsilon}(\mathrm{d} t, \mathrm{~d} x) \\
\leq & \lambda e^{\theta t} V_{1}^{\lambda-1}(\psi)\left\{-V_{1}(\psi)\left[r-\beta_{2}-\xi^{2} / 2-\int_{\mathbb{X}} \theta(x) \pi(\mathrm{d} x)-\lambda \xi^{2} / 2\right.\right. \\
& \left.\left.-\int_{\mathbb{X}}\left(\frac{1}{\lambda(1+\theta(x))^{\lambda}}-\frac{1}{\lambda}\right) \pi(\mathrm{d} x)-\theta / \lambda\right]+\beta_{1}\right\} \mathrm{d} t \\
& -\lambda \xi e^{\theta t} V_{1}^{\lambda}(\psi) \mathrm{d} B(t)+e^{\theta t} V_{1}^{\lambda}(\psi) \int_{\mathbb{X}}\left[\frac{1}{(1+\theta(x))^{\lambda}}-1\right] \widetilde{\Upsilon}(\mathrm{d} t, \mathrm{~d} x) \\
=: & e^{\theta t} \Psi(\psi) \mathrm{d} t-\lambda \xi e^{\theta t} V_{1}^{\lambda}(\psi) \mathrm{d} B(t)+e^{\theta t} V_{1}^{\lambda}(\psi) \int_{\mathbb{X}}\left[\frac{1}{(1+\theta(x))^{\lambda}}-1\right] \tilde{\Upsilon}(\mathrm{d} t, \mathrm{~d} x),
\end{aligned}
$$

where

$$
\begin{aligned}
\Psi(\psi)= & \lambda V_{1}^{\lambda-1}(\psi)\left\{-V_{1}(\psi)\left[r-\beta_{2}-\xi^{2} / 2-\int_{\mathbb{X}} \theta(x) \pi(\mathrm{d} x)\right.\right. \\
& \left.\left.-\lambda \xi^{2} / 2-\int_{\mathbb{X}}\left(\frac{1}{\lambda(1+\theta(x))^{\lambda}}-\frac{1}{\lambda}\right) \pi(\mathrm{d} x)-\theta / \lambda\right]+\beta_{1}\right\} .
\end{aligned}
$$

Then (11) implies that $C_{5}:=\sup _{\psi>0} \Psi(\psi)<+\infty$. Hence,

$$
\mathrm{d} V_{3}(t) \leq C_{5} e^{\theta t} \mathrm{~d} t-\lambda \xi e^{\theta t} V_{1}^{\lambda}(\psi) \mathrm{d} B(t)+e^{\theta t} V_{1}^{\lambda}(\psi) \int_{\mathbb{X}}\left[\frac{1}{(1+\theta(x))^{\lambda}}-1\right] \tilde{\Upsilon}(\mathrm{d} t, \mathrm{~d} x) .
$$

Therefore,

$$
\mathbb{E}\left[e^{\theta t} V_{1}^{\lambda}(\psi(t))\right] \leq V_{1}^{\lambda}(0)+C_{5}\left(e^{\theta t}-1\right) / \theta .
$$


Thereby,

$$
\limsup _{t \rightarrow+\infty} \mathbb{E}\left[\psi^{-\lambda}(t)\right] \leq C_{5} / \theta=: C_{6}
$$

For $\forall \varepsilon>0$, let $\kappa_{2}=\varepsilon^{1 / \lambda} / C_{6}^{1 / \lambda}$. Then Chebyshev's inequality indicates that

$$
\mathrm{P}\left\{\psi(t)<\kappa_{2}\right\}=\mathrm{P}\left\{\psi^{-\lambda}(t)>\kappa_{2}^{-\lambda}\right\} \leq \mathbb{E}\left[\psi^{-\lambda}(t)\right] / \kappa_{2}^{-\lambda}=\kappa_{2}^{\lambda} \mathbb{E}\left[\psi^{-\lambda}(t)\right]
$$

Thus limsup $\sup _{t \rightarrow+\infty} \mathrm{P}\left\{\psi(t)<\kappa_{2}\right\} \leq \varepsilon$. Consequently, (9) holds.

Now we study the extinction of model (3).

Theorem 3 If $\mu<0$ and $\beta_{1}>\beta_{2}^{2} \alpha$, then $\lim _{t \rightarrow+\infty} \psi(t)=0$ a.s.

Proof By Itô's formula, one has

$$
\begin{aligned}
\ln \psi( & t)-\ln \psi(0) \\
=r & -\frac{\xi^{2}}{2} t-\int_{0}^{t}\left[\beta_{1} \psi(s)+\frac{\beta_{2}}{\beta_{2} \alpha \psi(s)+1}\right] \mathrm{d} s+\xi B(t) \\
& +\int_{0}^{t} \int_{\mathbb{X}}\left(\ln \left(\psi\left(s^{-}\right)+\theta(x) \psi\left(s^{-}\right)\right)-\ln \left(\psi\left(s^{-}\right)\right)-\theta(x) \psi\left(s^{-}\right) \frac{1}{\psi\left(s^{-}\right)}\right) \pi(\mathrm{d} x) \mathrm{d} s \\
& +\int_{0}^{t} \int_{\mathbb{X}}\left(\ln \left(\psi\left(s^{-}\right)+\theta(x) \psi\left(s^{-}\right)\right)-\ln \left(\psi\left(s^{-}\right)\right)\right) \tilde{\Upsilon}(\mathrm{d} s, \mathrm{~d} x) \\
= & r t-\beta_{2} t-\frac{\xi^{2}}{2} t-\int_{0}^{t}\left[\beta_{1} \psi(s)-\frac{\beta_{2}^{2} \alpha \psi(s)}{\beta_{2} \alpha \psi(s)+1}\right] \mathrm{d} s+\xi B(t) \\
& -t \int_{\mathbb{X}}(\theta(x)-\ln (1+\theta(x))) \pi(\mathrm{d} x)+\int_{0}^{t} \int_{\mathbb{X}} \ln (1+\theta(x)) \tilde{\Upsilon}(\mathrm{d} s, \mathrm{~d} x) \\
= & \mu t-\int_{0}^{t}\left[\beta_{1} \psi(s)-\frac{\beta_{2}^{2} \alpha \psi(s)}{\beta_{2} \alpha \psi(s)+1}\right] \mathrm{d} s+\xi B(t)+Q(t),
\end{aligned}
$$

where

$$
Q(t)=\int_{0}^{t} \int_{\mathbb{X}} \ln (1+\theta(x)) \tilde{\Upsilon}(\mathrm{d} s, \mathrm{~d} x) .
$$

In view of Hypothesis $(\mathrm{H})$, we can derive that

$$
\langle Q, Q\rangle(t)=t \int_{\mathbb{X}}(\ln (1+\theta(x)))^{2} \pi(\mathrm{d} x)<C_{1} t
$$

Then the strong law of large numbers for local martingales (see [16]) indicates that

$$
\lim _{t \rightarrow+\infty} t^{-1} Q(t)=0 \quad \text { a.s. }
$$

In addition, notice that if $\beta_{1}>\beta_{2}^{2} \alpha$, then for $B \geq 0$,

$$
\beta_{1} \psi-\frac{\beta_{2}^{2} \alpha \psi}{\beta_{2} \alpha \psi+1} \geq \beta_{1} \psi-\beta_{2}^{2} \alpha \psi \geq 0
$$


Consequently,

$$
\limsup _{t \rightarrow+\infty} t^{-1} \ln \psi(t) \leq \mu<0
$$

Thus $\lim _{t \rightarrow+\infty} \psi(t)=0$ a.s.

\section{The growth rate}

Lemma 2 ([1, Theorem 5.2.9]) Suppose that $h_{1}:[0, \infty) \rightarrow \mathbb{R}$ and $h_{2}:[0, \infty) \times \mathbb{X} \rightarrow \mathbb{R}$ are two $\mathcal{F}_{t}$-adapted predictable stochastic processes. If for arbitrary $T>0$,

$$
\int_{0}^{T}\left|h_{1}(s)\right|^{2} \mathrm{~d} s<\infty, \quad \int_{0}^{T} \int_{\mathbb{X}}\left|h_{2}(x, s)\right|^{2} \pi(\mathrm{d} x) \mathrm{d} s<\infty,
$$

then for arbitrary constants $c_{1}>0$ and $c_{2}>0$,

$$
\begin{gathered}
\mathrm{P}\left\{\operatorname { s u p } _ { 0 \leq s \leq T } \left[\int_{0}^{s} h_{1}(v) \mathrm{d} B(v)-\frac{c_{1}}{2} \int_{0}^{t}\left|h_{1}(v)\right|^{2} \mathrm{~d} v+\int_{0}^{s} \int_{\mathbb{X}} h_{2}(x, v) \tilde{\Upsilon}(\mathrm{d} x, \mathrm{~d} v)\right.\right. \\
\left.\left.-\frac{1}{c_{1}} \int_{0}^{s} \int_{\mathbb{X}}\left(e^{c_{1} h_{2}(x, v)}-1-c_{1} h_{2}(x, v)\right) \pi(\mathrm{d} x) \mathrm{d} v\right]>c_{2}\right\} \leq e^{-c_{1} c_{2}} .
\end{gathered}
$$

Theorem 4 For Eq. (3), we have

$$
\limsup _{t \rightarrow+\infty} \frac{\ln \psi(t)}{\ln t} \leq 1 \quad \text { a.s. }
$$

Proof According to Itồs formula, we get

$$
\begin{aligned}
& e^{t} \ln \psi(t)-\ln \psi(0) \\
& =\int_{0}^{t} e^{s}\left[\ln \psi(s)+r-\frac{\xi^{2}}{2}-\beta_{1} \psi(s)-\frac{\beta_{2}}{\beta_{2} \alpha \psi(s)+1}\right] \mathrm{d} s \\
& \quad+\int_{0}^{t} \xi e^{s} \mathrm{~d} B(s)+\int_{0}^{t} \int_{\mathbb{X}} e^{s} \ln (1+\theta(x)) \tilde{\Upsilon}(\mathrm{d} s, \mathrm{~d} x) .
\end{aligned}
$$

Let $T=k \chi, c_{1}=\varepsilon e^{-k \chi}, c_{2}=\frac{\phi e^{k \chi} \ln k}{\varepsilon}$, where $i \in \mathbb{N}, \varepsilon \in(0,1), \chi>0$ and $\phi>1$, then Lemma 2 implies that

$$
\begin{aligned}
\mathrm{P}\left\{\operatorname { s u p } _ { 0 \leq s \leq T } \left[\int_{0}^{s} \xi e^{v} \mathrm{~d} B(v)-\frac{\varepsilon e^{-k \chi}}{2} \int_{0}^{s} \xi^{2} e^{2 v} \mathrm{~d} v+\int_{0}^{s} \int_{\mathbb{X}} e^{v} \ln (1+\theta(x)) \tilde{\Upsilon}(\mathrm{d} x, \mathrm{~d} v)\right.\right. \\
\left.\left.\quad-\frac{1}{\varepsilon e^{-k \chi}} \int_{0}^{s} \int_{\mathbb{X}}\left((1+\theta(x))^{\varepsilon e^{\nu-k \chi}}-1-\varepsilon e^{\nu-k \chi} \ln (1+\theta(x))\right) \pi(\mathrm{d} x) \mathrm{d} v\right]>\frac{\phi e^{k \chi} \ln k}{\varepsilon}\right\} \\
\leq k^{-\phi} .
\end{aligned}
$$

Notice that $\sum_{k=1}^{+\infty} k^{-\phi}<+\infty$, so the Borel-Cantelli lemma implies that there is an integer $\widehat{k}$ such that for arbitrary $k \geq \widehat{k}$ and $0 \leq t \leq k \chi$,

$$
\int_{0}^{t} \xi e^{v} \mathrm{~d} B(v)+\int_{0}^{t} \int_{\mathbb{X}} e^{v} \ln (1+\theta(x)) \tilde{\Upsilon}(\mathrm{d} x, \mathrm{~d} v)
$$




$$
\begin{aligned}
\leq & \frac{\phi e^{k \chi} \ln k}{\varepsilon}+\frac{\varepsilon e^{-k \chi}}{2} \int_{0}^{t} \xi^{2} e^{2 v} \mathrm{~d} v \\
& +\frac{1}{\varepsilon e^{-k \chi}} \int_{0}^{t} \int_{\mathbb{X}}\left((1+\theta(x))^{\varepsilon e^{\nu-k \chi}}-1-\varepsilon e^{\nu-k \chi} \ln (1+\theta(x))\right) \pi(\mathrm{d} x) \mathrm{d} \nu .
\end{aligned}
$$

It follows from (7) that

$$
(1+\theta(x))^{\varepsilon e^{\nu-k \chi}}-1-\varepsilon e^{\nu-k \chi} \ln (1+\theta(x)) \leq \varepsilon e^{\nu-k \chi}(\theta(x)-\ln (1+\theta(x))) .
$$

Making use of this inequality, (14) and (15) yield that for arbitrary $k \geq \widehat{k}$ and $0 \leq t \leq k \chi$,

$$
\begin{aligned}
& e^{t} \ln \psi(t)-\ln \psi(0) \\
& \leq \frac{\phi e^{k \chi} \ln k}{\varepsilon}+\int_{0}^{t} e^{s}\left[\ln \psi(s)+r-\frac{\xi^{2}}{2}-\beta_{1} \psi(s)-\frac{\beta_{2}}{\beta_{2} \alpha \psi(s)+1}\right] \mathrm{d} s \\
&+\frac{\varepsilon e^{-k \chi}}{2} \int_{0}^{t} \xi^{2} e^{2 s} \mathrm{~d} s+\left(e^{t}-1\right) \int_{\mathbb{X}}(\theta(x)-\ln (1+\theta(x))) \pi(\mathrm{d} x) \\
&= \frac{\phi e^{k \chi} \ln k}{\varepsilon}+\int_{0}^{t} e^{s}\left[\ln \psi(s)+r-\frac{\xi^{2}}{2}+\frac{\varepsilon \xi^{2} e^{s-k \chi}}{2}-\beta_{1} \psi(s)-\frac{\beta_{2}}{\beta_{2} \alpha \psi(s)+1}\right] \mathrm{d} s \\
&+\left(e^{t}-1\right) \int_{\mathbb{X}}(\theta(x)-\ln (1+\theta(x))) \pi(\mathrm{d} x) \\
& \leq \frac{\phi e^{k \chi} \ln k}{\varepsilon}+\int_{0}^{t} e^{s}\left[\ln \psi(s)+r-\frac{\xi^{2}}{2}+\frac{\xi^{2}}{2}-\beta_{1} \psi(s)\right] \mathrm{d} s \\
&+\left(e^{t}-1\right) \int_{\mathbb{X}}(\theta(x)-\ln (1+\theta(x))) \pi(\mathrm{d} x) \\
& \leq \frac{\phi e^{k \chi} \ln k}{\varepsilon}+\left(C_{7}+\int_{\mathbb{X}}(\theta(x)-\ln (1+\theta(x))) \pi(\mathrm{d} x)\right)\left(e^{t}-1\right) .
\end{aligned}
$$

In other words, for $k \geq \widehat{k}$ and $(k-1) \chi \leq t \leq k \chi$,

$$
\frac{\ln \psi(t)}{\ln t} \leq \frac{\ln \psi(0)}{e^{t} \ln t}+\frac{\phi e^{k \chi} \ln k}{e^{(k-1) \chi} \ln ((k-1) \chi) \varepsilon}+\left(C_{7}+\int_{\mathbb{X}}(\theta(x)-\ln (1+\theta(x))) \pi(\mathrm{d} x)\right) \frac{1-e^{-t}}{\ln t} .
$$

Letting $k \rightarrow+\infty$ results in

$$
\limsup _{t \rightarrow+\infty} \frac{\ln \psi(t)}{\ln t} \leq \frac{\phi e^{\chi}}{\varepsilon}
$$

Letting $\phi \downarrow 1, \chi \downarrow 0$, and $\varepsilon \uparrow 1$ gives the required assertion.

\section{Conclusions and simulations}

In this paper, we utilized a Lévy jump process to characterize the abrupt perturbations in the environment, and formulated a stochastic single species model with Allee effect and jump-diffusion. We proved that the model admits a unique solution which is global and positive, and investigated the stochastic permanence, extinction and the growth rate of the solution.

Out results reveal that the properties of the model have close relationships with the jump-diffusion. As a matter of fact, Theorem 2 illustrates that if the intensity of the jump 


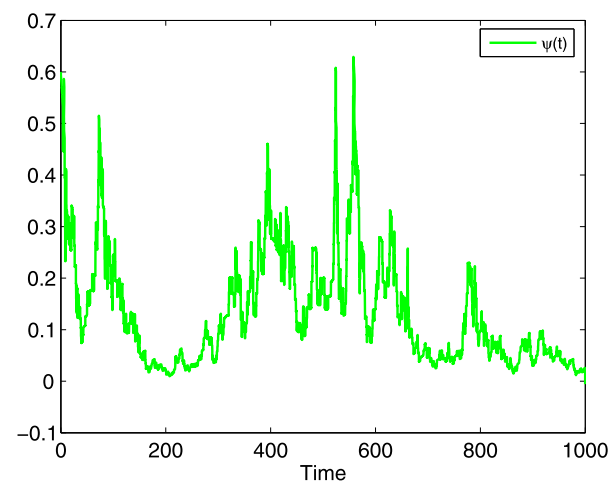

(a)

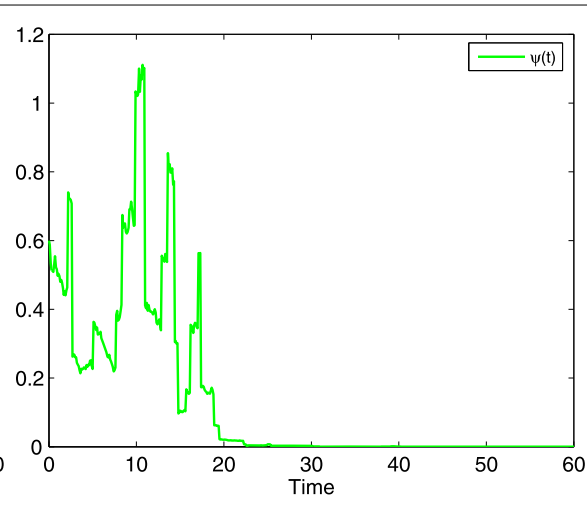

(b)

Figure 1 Solutions of Eq. (3) with $r=0.4, \beta_{1}=0.1, \beta_{2}=0.2, \alpha=0.3, \xi^{2}=0.2, \mathbb{X}=(0,+\infty), \pi(\mathbb{X})=1$, step size $\Delta t=0.001$ : (a) $\theta(x) \equiv 0.3504$, hence $\mu=0.05>0$. This figure shows that the species is SP; $(\mathbf{b}) \theta(x) \equiv 0.7722$, hence $\beta_{1}>\beta_{2}^{2} \alpha$ and $\mu=-0.1$. This figure shows that the species is extinctive. The numerical method is the Euler scheme in [22]. The parameter values are hypothetical

is sufficiently small such that

$$
\int_{\mathbb{X}}(\theta(x)-\ln (1+\theta(x))) \pi(\mathrm{d} x)<r-\frac{\xi^{2}}{2},
$$

then the species is SP (see Fig. 1(a)); Theorem 3 illustrates that if $\beta_{1}>\beta_{2}^{2} \alpha$ and the intensity of the jump is sufficiently large such that

$$
\int_{\mathbb{X}}(\theta(x)-\ln (1+\theta(x))) \pi(\mathrm{d} x)>r-\beta_{2}-\frac{\xi^{2}}{2},
$$

then the species is extinctive (see Fig. 1(b)).

Some problems related to these fields deserve further studies. First, there is a condition $\beta_{1}>\beta_{2}^{2} \alpha$ in Theorem 3. What happens if this condition is not satisfied? Second, it is interesting to consider other random perturbations, such as the telephone noise (see, e.g., $[17,18,24])$. When the telephone noise is considered, model (3) is replaced by:

$$
\begin{aligned}
\mathrm{d} \psi(t)= & \psi(t)\left(r(\gamma(t))-\beta_{1}(\gamma(t)) \psi(t)-\frac{\beta_{2}(\gamma(t)) \psi(t)}{\beta_{2}(\gamma(t)) \alpha(\gamma(t)) \psi(t)+1}\right) \mathrm{d} t \\
& +\xi(\gamma(t)) \psi(t) \mathrm{d} B(t)+\int_{\mathbb{X}} \theta(x, \gamma(t)) \psi(t) \tilde{\Upsilon}(\mathrm{d} t, \mathrm{~d} x)
\end{aligned}
$$

where $\gamma(t)$ is a continuous-time Markov chain with finite state. Finally, the present study supposes that only $r$ is perturbed by the white noise. It is of interest to test the case that other parameters, for instance, both $\beta_{1}$ and $\beta_{2}$, are also influenced. That is to say, to consider the following model:

$$
\begin{aligned}
\mathrm{d} \psi(t)= & \psi(t)\left(r-\beta_{1} \psi(t)-\frac{\beta_{2} \psi(t)}{\beta_{2} \alpha \psi(t)+1}\right) \mathrm{d} t+\xi_{1} \psi(t) \mathrm{d} B_{1}(t)+\xi_{2} \psi^{2}(t) \mathrm{d} B_{2}(t) \\
& +\frac{\xi_{3} \psi(t)}{\beta_{2} \alpha \psi(t)+1} \mathrm{~d} B_{3}(t)+\int_{\mathbb{X}} \theta(x) \psi(t) \tilde{\Upsilon}(\mathrm{d} t, \mathrm{~d} x),
\end{aligned}
$$

where $B_{1}(t), B_{2}(t)$ and $B_{3}(t)$ are standard Brownian motion. 
Acknowledgements

The author thanks the anonymous referee for his valuable comments.

\section{Funding}

Not applicable.

\section{Availability of data and materials}

Data sharing not applicable to this article as no datasets were generated or analyzed during the current study.

\section{Competing interests}

The author declares that they have no competing interests.

\section{Authors' contributions}

The single author is responsible for the complete manuscript. The author read and approved the final manuscript.

\section{Publisher's Note}

Springer Nature remains neutral with regard to jurisdictional claims in published maps and institutional affiliations.

Received: 3 October 2019 Accepted: 13 April 2020 Published online: 19 April 2020

\section{References}

1. Applebaum, D.: Lévy Processes and Stochastics Calculus, 2nd edn. Cambridge University Press, Cambridge (2009)

2. Bao, J., Mao, X., Yin, G., Yuan, C.: Competitive Lotka-Volterra population dynamics with jumps. Nonlinear Anal. 74, 6601-6616 (2011)

3. Bao, J., Yuan, C.: Stochastic population dynamics driven by Lévy noise. J. Math. Anal. Appl. 391, 363-375 (2012)

4. Berec, L., Angulo, E., Courchamp, F.: Multiple Allee effects and population management. Trends Ecol. Evol. 22, 185-191 (2007)

5. Boukal, D.S., Berec, L.: Single-species models of the Allee effect: extinction boundaries, sex ratios and mate encounters. J. Theor. Biol. 218, 375-394 (2002)

6. Clutton-Brock, T.H., Gaynor, D., Mcllrath, G.M., MacColl, A.D., et al.: Predation, group size and mortality in a cooperative mongoose, Suricata suricatta. J. Anim. Ecol. 68, 672-683 (1999)

7. Courchamp, F., Berec, J., Gascoigne, J.: Allee Effects in Ecology and Conservation. Oxford University Press, New York (2008)

8. Deng, M.: Dynamics of a stochastic population model with Allee effect and Lévy jumps. Physica A 531, 121745 (2019)

9. Dennis, B.: Allee effects: population growth, critical density, and the chance of extinction. Nat. Resour. Model. 3, 481-538 (1989)

10. Gopalsamy, K., Ladas, G.: On the oscillation and asymptotic behavior of $\dot{N}(t)=N(t)\left[a+b N(t-\tau)-c N^{2}(t-\tau)\right]$. Q. Appl. Math. 48, 433-440 (1990)

11. Gruntfest, Y., Arditi, R., Dombrovsky, Y.: A fragmented population in a varying environment. J. Theor. Biol. 185, 539-547 (1997)

12. Ji, W.: Permanence and extinction of a stochastic hybrid population model with Allee effect. Physica A 533, 122075 (2019)

13. Jovanović, M., Krstić, M.: The influence of time-dependent delay on behavior of stochastic population model with the Allee effect. Appl. Math. Model. 39, 733-746 (2015)

14. Kang, Y., Udiani, O.: Dynamics of a single species evolutionary model with Allee effects. J. Math. Anal. Appl. 418 492-515 (2014)

15. Kunita, H.: Itô's stochastic calculus: its surprising power for applications. Stoch. Process. Appl. 120, 622-652 (2010)

16. Lipster, R.: A strong law of large numbers for local martingales. Stochastics 3, 217-228 (1980)

17. Liu, M., Bai, C.: Optimal harvesting of a stochastic mutualism model with regime-switching. Appl. Math. Comput. 373 , $125040(2020)$

18. Liu, M., Deng, M.: Analysis of a stochastic hybrid population model with Allee effect. Appl. Math. Comput. 364, $124582(2020)$

19. Liu, M., Wang, K.: Stochastic Lotka-Volterra systems with Lévy noise. J. Math. Anal. Appl. 410, 750-763 (2014)

20. Liu, M., Zhu, Y.: Stationary distribution and ergodicity of a stochastic hybrid competition model with Lévy jumps. Nonlinear Anal. Hybrid Syst. 30, 225-239 (2018)

21. Mao, X.: Stochastic Differential Equations and Applications. Horwood, Chichester (1997)

22. Protter, P., Talay, D.: The Euler scheme for Lévy driven stochastic differential equations. Ann. Probab. 25, 393-423 (1997)

23. Stephens, P.A., Sutherland, W.J.: Consequences of the Allee effect for behaviour, ecology and conservation. Trends Ecol. Evol. 14, 401-405 (1999)

24. Wang, H., Liu, M.: Stationary distribution of a stochastic hybrid phytoplankton-zooplankton model with toxin-producing phytoplankton. Appl. Math. Lett. 101, 106077 (2020)

25. Wee, I.S.: Stability for multidimensional jump-diffusion processes. Stoch. Process. Appl. 80, 193-209 (1999)

26. Yu, X.W., Yuan, S.L., Zhang, T.H.: Persistence and ergodicity of a stochastic single species model with Allee effect under regime switching. Commun. Nonlinear Sci. Numer. Simul. 59, 359-374 (2018) 